RESEARCH ETHICS

\title{
Brief report on the experience of using proxy consent for incapacitated adults
}

\author{
S Mason, H Barrow, A Phillips, G Eddison, A Nelson, N Cullum, J Nixon
}

J Med Ethics 2006;32:61-62. doi: 10.1136/jme.2005.012302

See end of article for authors' affiliations

.....................

Correspondence to:

Su Mason, PhD, Clinical

Trials Research Unit,

University of Leeds, 17

Springfield Mount, Leeds,

LS2 9NG, UK; medsam@

leeds.ac.uk

Received 23 March 2005

In revised form

26 May 2005

Accepted for publication

8 June 2005
The Medicines for Human Use (Clinical Trials) Regulations 2004, which came into force in the UK in May 2004, cover the conduct of clinical trials on medicinal products. They allow a legal representative (a person not connected with the conduct of the trial) to consent to the participation of incompetent adults in medical research. Currently, very little is known about how such representatives will make their decisions. We have experience with proxy consent for older adults in a large, national trial. From 2445 potentially eligible but incapacitated patients, proxy, relative assent resulted in trial participation of only 87 (3.6\%) patients. The reasons for this were that a large number of incapacitated patients had no relative available for assent (2286), but also a high proportion of relatives approached refused to provide assent (72/159, 45.3\%). In comparison, $17.7 \%$ of patients declined participation in the trial.

Proxy consent allowed only a small increase in trial recruitment of incapacitated patients. The fact that a greater proportion of relatives than patients refused to provide assent implies that they were more cautious than the patients themselves, or perhaps used different criteria, when making their decision.

In future research involving incapacitated older patients there is likely to be heavy reliance on proxy consent provision by legal representatives. Our findings imply that consent decisions of legal representatives will not necessarily reflect those of patients themselves.
F or a valid informed consent to be given, ethical requirements are that: the consent is given voluntarily; the person giving consent has enough information to make an informed and reasoned choice; the person understands that information, and the person giving consent is mentally competent to give that consent. ${ }^{12}$

Overall, it is particularly in the area of incompetence (or lack of capacity) that older people may have problems in giving their consent to clinical trials (which involve the need to comprehend concepts such as randomisation, control groups, and benefit versus risk). ${ }^{3}$ Two thirds of acute hospital beds are occupied by people who are over 65 years and $40 \%$ of the overall National Health Service (NHS) budget is spent on older people (aged 65 years and over). ${ }^{4}$ More than a third of cancers are diagnosed in people over 75 , but this group is less extensively investigated and receives less treatment than younger patients. Indeed, very few studies include large numbers of old (over 75) or very old (over 85) people, ${ }^{5}$ resulting in lack of representation of this important group and thus lack of generalisability of research findings. ${ }^{67}$

The European Union Clinical Trials Directive (EC2001/20), which became transposed into UK law in May 2004, as the Medicines for Human Use (Clinical Trials) Regulations 2004, ${ }^{8}$ covers the conduct of clinical trials on medicinal products. The regulations include the provision of special protection to persons who are incapable of giving legal consent to clinical trials. ${ }^{9}$ They preclude inclusion of such people if the same results can be obtained using people who are able to provide consent and they state that the medicinal product should be of potential direct benefit to the patient, so that the benefits outweigh the risks.

The legislation allows a legal representative (who must be a person not connected with the conduct of the trial) to consent to the participation of incompetent adults in medical research. Depending on circumstances this may be either a "personal legal representative" (usually a close relative of the adult who lacks capacity), or, if such a person is not available, a "professional legal representative". The personal legal representative should be: a) suitable to act as the legal representative by virtue of their relationship with the adult, and b) available and willing to do so. The professional legal representative should be: a) the doctor primarily responsible for the adult's medical treatment, or b) a person nominated by the relevant healthcare provider.

In England and Wales such proxy consent has only formerly occurred with those who have parental responsibility (under the Children Act 1989) for medical care and research in children. Currently, very little is known about how such representatives will make their decisions-for example, whether they will accurately represent the incapacitated person's views.

We have experience of using proxy consent for older adults in a large NHS health technology assessment programme funded trial. This was the national, multicentre, "PRESSURE" trial (pressure relieving support surfaces: a randomised evaluation). Although this trial would not be covered by the Medicines for Human Use (Clinical Trials) Regulations 2004 (since the support surfaces under investigation were not a medicinal product), our data may be of interest to researchers who are planning studies that will involve consent on behalf of incapacitated patients by legal representatives.

\section{TRIAL AND PARTICIPANTS}

The PRESSURE trial ${ }^{10}$ aimed to determine whether there were differences between alternating pressure overlay mattresses and replacement mattresses, with respect to the development and healing of pressure ulcers, patient acceptability, and cost effectiveness. Patients were over 55 years, acute or elective, vascular, orthopaedic, medical or care of the elderly admissions, at high risk of pressure ulcer development due to mobility/activity limitation, or the presence of an existing pressure ulcer. Patients were consented, randomised,

Abbreviations: MREC, multicentre research ethics committee 
and allocated the trial mattress within 24 hours of admission to hospital.

Multicentre research ethics committee (MREC) approval was provided for "relative assent" by the patient's spouse or offspring for patients unable to provide informed consent for reasons including unconsciousness, semiconsciousness, and confusion. Such patients were deemed to be particularly at risk of developing a pressure ulcer and represented a group for whom the trial results would particularly apply.

\section{RESULTS}

Of 6155 patients assessed for eligibility, a total of 1972 patients were recruited and randomised to the PRESSURE trial between January 2001 and April 2004 from ten research centres. The median patient age was 76 years (range 55 to 100 years) and $63.9 \%$ patients were female.

There were 2445 potentially eligible, but incapacitated, patients from whom proxy, relative assent resulted in trial participation of only 87 (3.6\%) patients (median age 81 years, range 59 to 99 years). The main difficulties were the large number of incapacitated patients who had no relative available for assent (2286), and the high proportion of relatives approached who refused to provide assent (72/159, $45.3 \%)$.

In comparison, only $17.7 \%$ of patients approached declined participation in the trial (423 of the 2395 people approached to participate).

A protocol amendment was agreed by the MREC to expand the definition of relatives who were able to provide assent; from October 2002, this definition was expanded to those who were both a relative of the patient and the named next of kin (as recorded in the hospital or nursing notes). The following relatives provided assent: daughter (48); wife (13); son (13); husband (10); granddaughter (1); brother (1), and daughter in law (1). Thus the protocol amendment resulted in only three additional patients being recruited to the trial through assent from a granddaughter, brother, and daughter in law.

Of these 87 patients, eight subsequently regained capacity and were also able to provide consent for trial participation themselves.

\section{COMMENT}

This paper reports on the proxy assent in a single trial; however, the representativeness of the data is enhanced by the fact that it was a national, large, multicentre study. We do not know whether our findings are replicated in other trials involving incapacitated adults.

We found that the proxy consent process allowed only a small increase in trial recruitment of incapacitated patients, but arguably maintained respect for these patients and their family autonomy.

The fact that a greater proportion of relatives than patients refused to provide assent implies that they were more cautious (even for such a non-invasive trial) than the patients themselves, or perhaps used different criteria, or weighted these criteria differently from patients, when making their decision. It might be, however, that they found decision making on behalf of another adult too much of a burden. Certainly relatives appear to have thought carefully about the advantages and disadvantages of participation in the trial and did not liberally apply proxy altruism-(that is, many did not make altruistic decisions on behalf of their relative to enter into the trial.

In future research involving incapacitated older patients there is likely to be heavy reliance on proxy consent provision by legal representatives. Our findings imply that consent decisions of personal legal representatives do not necessarily reflect those of patients themselves. The findings also underline the concerns voiced about the ethics of the use of professional legal representatives, who are unlikely to know the previous will of the incapacitated adults whom they represent. ${ }^{11}$

The recent introduction of the Medicines for Human Use (Clinical Trials) Regulations 2004, the debate surrounding the Mental Capacity Act, ${ }^{12}$ and the ethical concerns about professional legal representatives render it timely and important for further research to examine the validity of consent by legal representatives. There is a need to establish whether the use of legal representatives (both personal and professional) is problematic. Qualitative research exploring the decisions made by legal representatives and the views of older people is needed to investigate whether proxy consent decisions are valid, congruent, and appropriate. It might be that representatives are more or less likely to be moved to consent by a type of altruism than those they represent. Or it might be that personal proxies try to apply a best interest test, but use processes that are less reliable than those currently used by healthcare professionals for patients under their care. Alternatively, proxies may turn out to be very good at giving valid consent that accurately represents the participants' interests and/or beliefs. Such a finding might reassure researchers, legal representatives, and elderly people, and lead to increased recruitment of elderly people into trials. Conversely, a poor match between proxy consent decisions and standard ones might raise doubts as to the purpose of proxy consent and whether it should have the power of standard consent.

\section{ACKNOWLEDGEMENTS}

This study was supported by a grant from the UK National Health Service Research and Development Health and Technology Assessment Programme. The views and opinions in the paper do not necessarily reflect those of the NHS Executive.

The authors would like to acknowledge the contribution of Peter Allmark, Faculty of Medicine, University of Sheffield, UK

\section{Authors' affiliations}

S Mason, A Phillips, G Eddison, J Nixon, Clinical Trials Research Unit, University of Leeds, Leeds, UK

H Barrow, Leeds Teaching Hospitals NHS Trust, Leeds, UK

A Nelson, N Cullum, Department of Health Sciences, University of York, York, UK

All authors declare no competing interests.

There has been substantial contribution by all authors to the: 1) conception and design, or acquisition of data, or analysis and interpretation of data; 2) drafting the article or revising it critically for important intellectual content, and 3) final approval of the version to be published.

\section{REFERENCES}

1 Beauchamp T, Childress J. Principles of biomedical ethics [5th ed]. Oxford: OUP, 2001.

2 Neuberger J. Ethics and health care. The role of research ethics committees in the United Kingdom. London: King's Fund Institute, 1992.

3 Sugarman J, McCory D, Hubal R. Getting meaningful informed consent from older adults: a structured literature review of empirical research. J Am Geriatrics Society 1998;46:517-24.

4 Department of Health. National service framework for older people. London: Department of Health, 2001.

5 Turner NJ, Haward RA, Mulley GP, et al. Cancer in old age-is it adequately investigated and treated? BMJ 1999;319:309-12.

6 Ferguson P. Selecting participants when testing new drugs: the implications of age and gender discrimination. Med Leg J 2002;70:130-4.

7 Weijer C. Selecting subjects for participation in clinical research: one sphere of justice. J Med Ethics 1999;25:31-6.

8 www.opsi.gov.uk/si/si2004/20041031.htm (accessed 17 Jul 2005)

9 www.corec.org.uk/recs/guidance/docs/ Informed_Consent_in_CTIMPs_(v1).doc (accessed 17 Jul 2005).

10 Nelson EA, Nixon J, Mason S, et al. A nurse led randomised trial of pressure relieving support surfaces. Prof Nurs 2003;18:513-16.

11 Nicholson R. Another threat to research in the United Kingdom. BMJ 2004;328:1212-13.

12 www.opsi.gov.uk/acts/acts2005/20050009.htm (accessed 17 Jul 2005). 